\title{
Software for scoring caries factors
}

Cariogram is a software program which aims to demonstrate the multifactorial background of dental caries by illustrating the interaction of caries-related factors. Patients are scored on diet, plaque, caries experience and saliva secretion, and the results shown on a pie chart. Cariogram was devised by Professor Douglas Bratthall at the University of Malmo, initially as an educational program.

The program may be used, it is suggested, as being able to illustrate the chances of the patient developing caries in the coming year. The chart has five sections, which reflect diet, bacteria, susceptibility, circumstances and the likelihood of developing caries. However, although fairly strong associations have been found between caries experience and these factors, it does not mean that these factors can successfully predict future caries experience. In fact Professor Bratthall stresses that while Cariogram may act as a guide to caries risk, it never specifies the number of cavities which may occur. The software will also produce some suggested measures for the patient to implement in order to reduce or even eliminate their risk.

The susceptibility category takes into account how much fluoride the patient's teeth are exposed to as well as saliva secretion and saliva buffer activity. The circumstances category looks at a combination of past caries

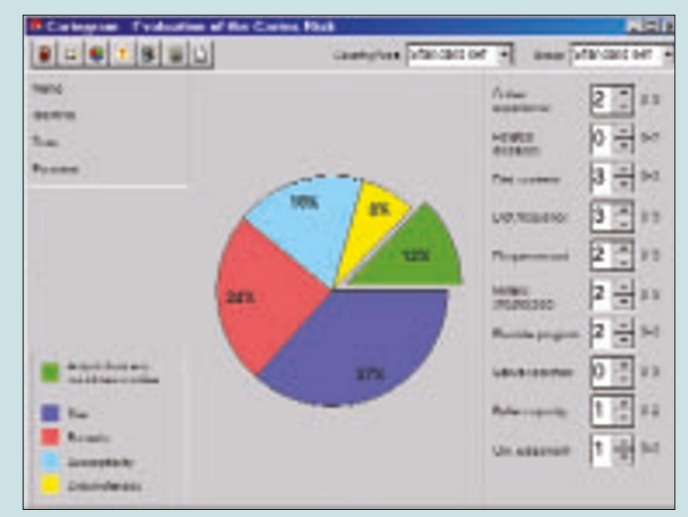

Cariogram chart showing high risk of caries

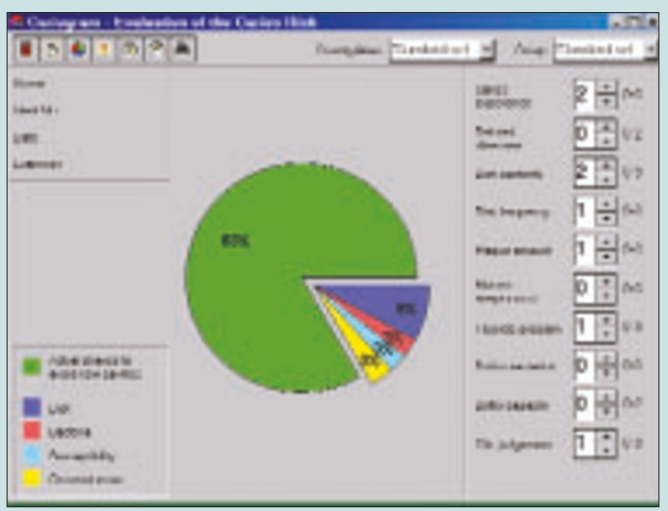

Cariogram chart showing low risk of caries experience and related diseases. These are balanced with diet and bacteria to produce the percentages.

Professor Bratthall believes that Cariogram's main principles will hold true in other countries although some of the factors which influence the scoring may vary. In these circumstances, practitioners are able to use their own clinical judgement to influence the outcome of the Cariogram. However, since the simulation model depends on salivary buffering and mutans tests which are not widely used in the UK, take-up of the software may not be so widespread as in Sweden. Its application is most likely to be in private practice on younger patients.

Professor Bratthall is program manager for the World Health Organisation oral database. He said his work on Cariogram began after he wrote to oral health experts around the world asking them to fill in a questionnaire on the factors which most influence caries reduction. Some thought it was better brushing, others thought it was better diet or use of fluoride. He decided he wanted to find a way of explaining all the different factors affecting caries and improved resistance to caries and how they relate to each other. Cariogram was produced in Sweden in 1997 and is now available in the UK through the British Dental Health Foundation. 